\title{
Negative affect subtypes and craving differentially predict long-term cessation success among smokers achieving initial abstinence
}

\author{
Yantao Zuo $^{1}$ • Norka E. Rabinovich ${ }^{2}$ • David G. Gilbert ${ }^{2}$
}

Received: 3 April 2016 / Accepted: 12 December 2016/Published online: 27 December 2016

(C) Springer-Verlag Berlin Heidelberg 2016

\begin{abstract}
Objective This study aimed to examine the associations of individual trajectories of three types of negative affect (NA: anxiety, depression, and anger) and craving during a 44-day period of incentivized smoking abstinence period with cessation outcome at 3 months and at 1 year.

Methods Adult smokers $(N=140)$ completed questionnaire assessments of NA and craving during pre-quit baseline sessions and 15 postquit sessions over the 45 days of biochemically verified abstinence while on nicotine or placebo patch treatment. Growth curve and logistic regression analyses were used to examine the associations of trajectory parameters of the individual NA states and craving with the abstinence outcomes at 3 months and 1 year postquit.

Results Greater declines in anxiety, depression, and anger symptoms over the first 44 days of smoking cessation were predictive of higher odds of abstinence at both 3 months and 1 year. Moreover, the greater declines in anxiety and anger remained as significant predictors of abstinence at both time
\end{abstract}

Electronic supplementary material The online version of this article (doi:10.1007/s00213-016-4509-1) contains supplementary material, which is available to authorized users.

David G. Gilbert

dgilbert@siu.edu

Yantao Zuo

yantao.zuo@duke.edu

Norka E. Rabinovich

norkar@siu.edu

1 Department of Psychiatry and Behavioral Sciences, Duke University Medical Center, Durham, NC 27705, USA

2 Department of Psychology, Southern Illinois University, Mailcode 6502, Carbondale, IL 62901, USA points, independent of the predictive ability of the trajectory profiles of craving.

Conclusions The findings suggest that slower dissipation of NA, especially anxiety and anger, represents a greater risk for relapse to smoking beyond that predicted by craving during early abstinence. Thus, temporal profiles of the affective symptoms convey unique motivational significance in relapse. Reduction in NA during early abstinence may be a valid target for interventions to increase long-term cessation success rates particularly among individuals with refractory affective symptoms.

Keywords Tobacco abstinence symptoms $\cdot$ Relapse ·

Negative affect · Craving · Growth curve analysis · Relapse

\section{Introduction}

Despite the advances in pharmacological treatments, most smokers undergo cessation treatment relapse within a year and they typically have to go through multiple attempts before they can achieve long-term abstinence (Borland et al. 2012). Understanding factors contributing to relapse proneness is thus of important theoretical and clinical significance. Relief of aversive (primarily increased negative affect) abstinence experiences is thought to both maintain tobacco dependence and lead to relapse (e.g., Baker et al. 2004; Solomon 1977). However, evidence for the relationship between abstinence symptoms and relapse has been limited (cf. McCarthy et al. 2006; Piasecki et al. 2000; Patten and Martin 1996). Possible reasons are that early studies combined multiple withdrawal symptoms into a single index relied on single-occasion measurements that fail to capture the dynamic dimensions of symptoms over time or failed to examine individual 
differences in dynamic patterns of symptoms (Hughes 2007; Piasecki et al. 2000).

Several recent studies have demonstrated robust individual differences in early tobacco abstinence symptoms which predict subsequent smoking relapse or lapses (Cofta-Woerpel et al. 2011; Javitz et al. 2012; McCarthy et al. 2006; Piasecki et al. 2000; Piasecki et al. 2003a,b; Piper et al. 2011). More specifically, Piasecki et al. (2003b) have presented evidence supporting their view that heightened risk for relapse is related to: symptom trajectory parameters that index more severe and refractory withdrawal symptoms, including: (1) greater average symptom severity, (2) abstinence symptom slopes indicating worsening of or slower reductions of symptoms over time, (3) curvature indicating unusual patterns such as a renewal of symptoms, and (4) volatility (unsystematic symptom scatter). Consistent with their suggestions, trajectory parameters of tobacco abstinence symptoms have been found to be substantially independent predictors of relapse (Javitz et al. 2012; Piasecki, 2006; Piasecki et al. 2003a,b) and likely reflect different neurobiological, temperamental, and environmental factors (Gilbert et al. 2009; Zuo et al. 2009). For example, one could hypothesize that volatility could relate to environmental factors, while slope might be expected to relate to neurobiological recovery from chronic nicotine exposure, and mean level may relate to genetically based or trait-like individual differences and/or degree of exposure-related neuroadaptation.

Though negative affect (NA) is a central component of tobacco abstinence (Baker et al. 2004), studies have reported mixed results with respect to whether NA uniquely predicts relapse among smokers receiving cessation treatment after controlling for craving, another defining feature of tobacco abstinence (Baker et al. 2012; Hughes and Hatsukami 1986; Shiffman 1979; Welsch et al. 1999). In a randomized placebocontrolled smoking cessation trial, Piper et al. (2011) found that increases in mean self-ratings of global NA and craving over 10 days postquit each accounted for unique variance in predicting relapse at 8 weeks postquit. Slower reductions or atypical temporal profiles of global NA and craving following cessation were also independently predictive of higher relapse risk at 30 days postquit (Piasecki et al. 2000). In contrast, Javitz et al. (2012) found that craving trajectories (average level, slope, and volatility) were predictive of both the shortterm and long-term relapse, whereas profiles of NA and other withdrawal symptoms contributed little to the withdrawal-relapse association. These inconsistent findings across studies raise the need to further evaluate the motivational significance of tobacco abstinence-related NA in relation to relapse. Also, it is not yet clear whether early symptoms predict relapse across a full year when early relapse is minimized by strong incentives for abstinence. Furthermore, although singleoccasion measurements of increase in anger (Castro et al. 2011; Patterson et al. 2008), anxiety (Castro et al. 2011;
Daughton et al. 1990), and depression (Cook et al. 2010; Hughes 1992; Etter and Hughes 2006; Leventhal et al. 2008) have been shown to predict relapse in some studies (but see Hughes 2007), it is not known whether trajectories of these distinct components of post-cessation NA are differentially linked to the risk for relapse.

The primary aim of the present analysis was to assess whether trajectories of major NA components (anger, anxiety, and depression) of affective symptoms during the first 44 days of smoking cessation predict later relapse to smoking. We examined these symptoms independently of the severity of cigarette craving and expanded extant research by measuring cessation at both short-term ( 3 months) and long-term ( 1 year) postquit. We hypothesized that more severe and refractory affective symptoms (e.g., higher average levels, slower rates of decrease, and greater volatility) would be associated with poorer abstinence outcomes, even after controlling for craving.

\section{Methods}

\section{Participants}

Data for this analysis were obtained from a clinical study, as described in detail by Gilbert et al. (2009). Upon completion of a 2.5 -week baseline phase, 169 dependent smokers who wanted to quit smoking were randomly assigned to the nicotine (NP) or placebo patch (PP) group in a double-blind manner (Fig. S1 in Supplementary Materials). A financial incentive (\$500) was used to maximize abstinence and to reduce the impact of dropouts on estimation of abstinence symptoms during the 45-day treatment phase, but not the follow-up phase. Biochemically verified continuous abstinence and study compliance during the 45-day contingency for abstinence period were maintained by $73 / 89(82 \%)$ in the NP group and 67/80 (84\%) in the PP group, defined as smoking four or fewer cigarettes total across the 45-day treatment period, and having a breath $\mathrm{CO}$ of $<8 \mathrm{ppm}$, salivary cotinine $(<20 \mathrm{ng} / \mathrm{ml})$, and plasma nicotine $<1.5 \mathrm{ng} / \mathrm{ml}$. Data from these participants $(N=140)$ were focused on in the present analysis.

\section{Procedure}

Prior to study enrollment, participants signed an informed consent approved by the Southern Illinois University Human Subjects Committee. There were three study phases. Phase 1 was a 2.5 -week baseline period, during which participants smoked at their usual rate and attended five monitoring sessions with at least 2 days in between each session. Phase 2 included afternoon monitoring sessions on Quit Day (C1), Day 3 of abstinence (C3), then every $72 \mathrm{~h}$ (C6 to C15), on Day 17 (C17), and then every $72 \mathrm{~h}(\mathrm{C} 20$ to $\mathrm{C} 44)$. At each 
monitor session, participants were asked to rate their average levels of craving for cigarettes and mood states over the past $72 \mathrm{~h}$ or since the last visit. The present analysis excludes data from the first $(\mathrm{C} 1)$ and the final day of required abstinence (Day 45) because the former was subject to residual effects of smoking and initial reactions to nicotine or placebo patch, while the latter was devoted to a lengthy experimental session. Phase 3 was the post-abstinence contingency/post-treatment follow-up period that is the focus of this report.

\section{Financial contingencies}

Completion of all aspects of study phases 1 and 2 resulted in earning \$500 minus any penalties for smoking that was provided a few weeks after the completion of phase 2 . Penalties for the first to the third cigarette were $\$ 10, \$ 25$, and $\$ 50$, respectively, with a maximum total of $\$ 85$ for three or four cigarettes. Participants were excluded from the study without pay if they smoked more than four cigarettes total across the 45-day phase-2 period, but were free to return to smoking or not during the 3,6, 9, and 12-month follow-up sessions for which they received additional compensation $(\$ 25, \$ 25, \$ 25$, and \$50) for completing timeline followback questionnaires that assessed daily smoking amounts during the preceding 3 months.

Participants received an abbreviated form of the American Lung Association smoking cessation program. For individuals in the NP group, NicoDerm CQ® patches were $21 \mathrm{mg}$ for the first 17 days of abstinence (Sessions $\mathrm{C} 1$ to $\mathrm{C} 17), 14 \mathrm{mg}$ for days 18-26 (C20 to C26), and $7 \mathrm{mg}$ for days 27-38 (C29 to C38). Subjects in PP group received placebo patches for 38 days. The final assessment of abstinence symptoms 1 week after going off the patch was chosen to characterize rebound effects (see Supplementary Materials and Gilbert et al. 2009 for more details).

\section{Questionnaires}

The Fagerström Test of Nicotine Dependence (FTND; Heatherton et al. 1991), a widely used and validated measure of nicotine dependence, was administered at screening to assess nicotine dependence.

The Shiffman Withdrawal Questionnaire (SWQ; Shiffman 1979) was used to assess craving for cigarettes and other abstinence symptoms at each monitor session. Only scores on the craving subscale of the SWQ were analyzed in this report.

The Profile of Mood States (POMS; McNair et al. 1971) was used to assess mood states during monitor sessions. Subscale scores for tension-anxiety, anger, and depression were analyzed in this report.

\section{Determination of smoking/abstinence status}

The number of cigarettes smoked during the first 45-day quit period was estimated by a combination of 3-day self-report time-line followback, carbon monoxide (CO) concentration ( $8+\mathrm{ppm})$, and (for PP group only) plasma or salivary cotinine $(20 \mathrm{ng} / \mathrm{ml})$, as well as plasma nicotine $(1.5 \mathrm{ng} / \mathrm{ml})$ and by knowledge of the half-lives of these substances. $\mathrm{CO}$ and salivary samples were obtained at every monitoring session (every $72 \mathrm{~h}$ or less) during the postquit period. Cotinine and nicotine concentrations were assayed, as previously described (Jacob et al. 1981). Nicotine and cotinine concentrations were determined from blood samples collected during the third prequit session and then at days 1, 3, 10, 17, and 45 after quitting. As reported previously (Gilbert et al. 2009), plasma nicotine and cotinine concentrations while on 21-mg patch were slightly higher than prequit levels among the abstainers in the NP group, but these values fell to nearly zero at the end of 1 week after going off the patch. Nicotine values in the PP group approximated zero after quitting. The $\mathrm{CO}$, cotinine, and self-report assessments confirmed good compliance with patch use in both groups during the incentive-motivated 45day abstinence period. Smoking relapse time-line followback estimates were obtained during assessments at 3, 6, 9, and 12 months following the quit day. In addition, approximately $80 \%$ of these follow-up assessments included in-lab assessments of $\mathrm{CO}$ concentrations in those who claimed abstinence. Relapse during the phase 3 follow-up assessments was defined as reporting smoking a single puff on two or more on consecutive days or $\mathrm{CO}$ concentrations of $8+\mathrm{ppm}$. All subjects who meet this criterion resumed daily smoking immediately after the second lapse day. Using these criteria, 52 subjects were classified as abstainers at 3 months. In comparison, 50 of them were identified as abstainers using the more common 7-day point prevalence criterion for abstinence (i.e., complete abstinence for at least 7 days prior to assessment; Hughes et al. 2010). For classification of abstaining vs. relapsing cases at 6 and 9 months and 1 year, using these two criteria produced identical results.

\section{Data analysis}

Data analysis mainly consisted of parameter estimation for person-specific trajectories of POMS anger, anxiety, and depression scores and SWQ craving scores across the first 44 days of abstinence and subsequent logistic regression analyses which determined the predictive associations of the trajectory parameters of affective and craving change with later abstinence status. A similar approach has been used by Javitz et al. (2012). Specifically, change scores of postquit NA states and craving measures for each individual at each postquit time point were generated by subtracting the average of the final two (fourth and fifth) prequit baseline session (B4 and B5) 
scores for that measure from each of the post-quit raw scores and then dividing the differences by the mean baseline standard deviation across individuals for the measure. For each of the four symptom scales, hierarchical linear modeling (HLM; Raudenbush and Bryk 2002) was used to estimate parameters representing level (centered at day 22.5 postquit), slope, and quadratic curvature of trajectory of the change scores. For each of the three parameters, a person-specific value was the sum of the estimated population average and person-specific variability (i.e., random effects). A person-specific volatility parameter was also calculated as the root mean square differences between the observed and predicted symptom scale scores.

Separate stepwise logistic regressions were first performed with abstinence at 3 months and 1 year postquit as dependent variables and person-specific trajectory parameters for each symptom component as the independent variables to determine their predictive associations. Gender, age, FTND score, and treatment (NP vs. PP) are entered as controlled variables in the regressions. An additional controlled variable was the baseline measurement of that particular symptom component, defined as the mean of standardized scores of the final two baseline sessions. To evaluate the extent to which the trajectory parameters of an affective symptom component contribute unique predictive utility beyond that accounted by those of craving, joint regressions were conducted to include both sets of parameters, provided each set was shown to contain at least one significant predictor of abstinence. The levels of statistical significance for variable entry and removal were set at 0.05 and 0.10 , respectively. Abstinence at both 3 months and 1 year postquit was assessed because proximity to cessation outcome is a significant factor with respect to withdrawal-relapse relation (Wray et al. 2013). A cutting point for short vs. long abstinence in the study sample was empirically determined from the baseline survival curve. There was a rapid drop in abstinence rate between EOT and 3 months postquit followed by a slower decline afterwards (Fig. S2 in Supplementary Materials). Therefore, abstinence at 3 months was chosen to represent short-term cessation outcome. In addition, similar regression analyses were conducted to evaluate the predictive associations of trajectory parameters of affective symptoms and craving with abstinence status at two intermediate time points (6 and 9 months).

To further demonstrate the association of temporal trajectories of distinct symptom components and abstinence, a 2-level HLM model was fitted with Level 1 submodel representing the trajectory of each symptom component and Level 2 submodel including abstinence as a predictor. All 2-level models were fitted using full maximum likelihood method and parameter estimates based on robust standard errors. Based on the estimated parameters, predicted trajectories for each symptom component were constructed to visualize group differences (abstinence vs. relapse) in temporal profiles of that component.

\section{Results}

\section{Sample characteristics}

Table 1 summarizes demographic and baseline smokingrelated characteristics and the abstinence rates of the subjects by treatment groups. They were mostly young adults (mean age $=26.1)$, Caucasian $(91.4 \%)$, and smokers of both sexes (49.3\% men). On average, they smoked 18.9 cigarettes per day (CPD) and scored 4.6 on the FTND. Nicotine and placebo patch groups did not differ in terms of demographical, baseline smoking heaviness, and abstinence rates at 3, 6, 9 months and 1 year postquit ( $t$ test for continuous variables and $\chi^{2}$ for categorical variables; all $p s>.15)$.

\section{Correlations between trajectory parameters}

The Pearson correlations of trajectory parameters within and across symptom components are presented in Supplemental Table S1. Within each symptom component, the correlations between trajectory parameters ranged from -0.56 to 0.40 . There were no systematic patterns in these correlations across all symptom components except consistent, modest correlations between trajectory level, and volatility across the three affective symptom components $(0.21$ to 0.40$)$, but not for craving (0.03). Correlations of trajectory parameters of the affective components with the corresponding parameters of craving were mostly modest $(0.10$ to 0.42$)$, while those between the affective components were in the medium to moderately high range $(0.41$ to 0.77$)$. These results indicated that each trajectory parameter represented a largely distinct aspect of symptom dynamics, and the correlations of the corresponding parameters tended to be higher between the affective components than between them and craving.

Table 1 Descriptives of subjects in nicotine and placebo patch groups

\begin{tabular}{|c|c|c|c|}
\hline & $\begin{array}{l}\text { Nicotine patch } \\
(n=73)\end{array}$ & $\begin{array}{l}\text { Placebo patch } \\
(n=67)\end{array}$ & $\begin{array}{l}\text { Total } \\
(n=140)\end{array}$ \\
\hline Gender (male) & $34(46.6 \%)$ & $35(52.2 \%)$ & $69(49.3 \%)$ \\
\hline Ethnicity (Caucasian) & $65(89.0 \%)$ & $63(94.0 \%)$ & $128(91.4 \%)$ \\
\hline Age & $27.0(8.6)$ & $25.2(7.3)$ & $26.1(8.1)$ \\
\hline FTND & $4.9(2.1)$ & $4.4(2.2)$ & $4.6(2.2)$ \\
\hline Prequit CPD & $19.1(6.8)$ & $18.7(5.4)$ & $18.9(6.1)$ \\
\hline Pack-year & $11.1(10.5)$ & $9.1(8.1)$ & $10.2(9.5)$ \\
\hline \multicolumn{4}{|l|}{ Abstinent cases at } \\
\hline Postquit 3 months & $28(38.4 \%)$ & $24(35.8 \%)$ & $52(37.1 \%)$ \\
\hline Postquit 6 months & $21(28.8 \%)$ & $13(19.4 \%)$ & $34(24.3 \%)$ \\
\hline Postquit 9 months & $11(15.1 \%)$ & $11(16.4 \%)$ & $22(15.7 \%)$ \\
\hline Postquit 1 year & $10(13.7 \%)$ & $9(13.4 \%)$ & $19(13.6 \%)$ \\
\hline
\end{tabular}

Mean $( \pm \mathrm{SD})$ are reported for continuous variables FTND Fagerström Test of Nicotine Dependence, $C P D$ cigarettes smoked per day 


\section{Prediction of abstinence at 3 months}

The top section of Table 2 shows the odds ratios of abstinence at 3 months postquit as significantly predicted by trajectory parameters of a single symptom component in logistic regression analysis controlling the effects of gender, age, FTND score, treatment, and baseline measurement of that symptom component. The left panels in Figs. 1 and 2 depict, respectively, the group average trajectories and volatility of each symptom component in the abstainers and relapsers at 3 months. Among the four trajectory parameters for changes in the postcessation POMS tension-anxiety subscale score, only the slope was found to be a significant predictor of abstinence at 3 months. Greater negative slope values of the tension-anxiety trajectory, which represented smaller decreases over the first 44 days postquit, were associated with lower odds of abstinence at 3 months ( $p=0.005$; Fig. 1a). Conversely, larger decreases in tension-anxiety symptoms were linked to greater probability of abstinence. Similar results were found with the values of slope for the trajectories of POMS depression $(p=0.054)$ and anger subscale scores $(p=0.001)$. Greater values of curvature in the depression $(p=0.074)$ and anger symptom trajectories ( $p=0.097)$ were also marginally predictive of increased odds of abstinence. Thus, greater decline from initial rise in either symptom component was associated with higher odds of 3-month abstinence (Fig. 1c, e). In contrast, as reflected in both rising slope and slight concave curvature, mean post-cessation levels of depressive symptoms showed no initial spike and a trend for a continued elevation in depression over the 6-week treatment period among those who relapsed within 3 months postquit (Fig. 1c). Among these individuals, mean anger scores also remained nearly flat across the treatment period (Fig. 1e). In addition, higher levels of anger were predictive of lower odds of 3-month abstinence ( $p=0.001$; Fig. 1e). Greater volatility of changes in postcessation anger $(p=0.004)$ and, to a marginally significant level, depression scores $(p=0.052)$ were also related to greater odds of 3-month abstinence (Figs. 1c, e and 2c, e).

Table 2 Relationships between withdrawal symptom trajectory parameters and abstinence at 3 months postquit in stepwise logistic regressions

\begin{tabular}{|c|c|c|c|c|c|c|c|c|c|c|c|c|}
\hline & \multicolumn{3}{|c|}{ POMS Tension-anxiety } & \multicolumn{3}{|c|}{ POMS depression } & \multicolumn{2}{|c|}{ POMS anger } & \multicolumn{4}{|c|}{ SWQ craving } \\
\hline & $\begin{array}{l}\text { Odds } \\
\text { ratio }\end{array}$ & $\begin{array}{l}95 \% \text { C.I. } \\
\text { or odds ratio }\end{array}$ & $\begin{array}{l}p \\
\text { value }\end{array}$ & $\begin{array}{l}\text { Odds } \\
\text { ratio }\end{array}$ & $\begin{array}{l}95 \% \text { C.I. } \\
\text { for odds ratio }\end{array}$ & $\begin{array}{l}p \\
\text { value }\end{array}$ & $\begin{array}{l}\text { Odds } \\
\text { ratio }\end{array}$ & $\begin{array}{l}95 \% \text { C.I. } \\
\text { for odds ratio }\end{array}$ & $\begin{array}{l}p \\
\text { value }\end{array}$ & $\begin{array}{l}\text { Odds } \\
\text { ratio }\end{array}$ & $\begin{array}{l}95 \% \text { C.I. } \\
\text { for odds ratio }\end{array}$ & $\begin{array}{l}p \\
\text { value }\end{array}$ \\
\hline \multicolumn{13}{|c|}{ Single-component regression ${ }^{\mathrm{a}}$} \\
\hline Level & & & & & & & 0.45 & {$[0.28,0.74]$} & 0.001 & 0.72 & {$[0.50,1.03]$} & 0.070 \\
\hline Slope & 0.58 & {$[0.39,0.85]$} & 0.005 & 0.67 & {$[0.44,1.01]$} & 0.054 & 0.43 & {$[0.26,0.70]$} & 0.001 & & & \\
\hline Curvature & & & & 1.42 & {$[0.97,2.09]$} & 0.074 & 1.47 & {$[0.93,2.30]$} & 0.097 & & & \\
\hline Volatility & & & & 1.46 & {$[1.00,2.15]$} & 0.052 & 2.00 & {$[1.24,3.23]$} & 0.004 & & & \\
\hline $\begin{array}{l}\text { Baseline symptom } \\
\text { level }\end{array}$ & 1.82 & {$[1.22,2.73]$} & 0.003 & & & & & & & & & \\
\hline Age & & & & & & & 1.05 & {$[1.00,1.10]$} & 0.059 & & & \\
\hline FTND & & & & & & & 0.84 & {$[0.69,1.02]$} & 0.074 & & & \\
\hline \multicolumn{13}{|c|}{ Joint analysis regression $^{\mathrm{b}}$} \\
\hline \multicolumn{13}{|l|}{ Affect } \\
\hline \multicolumn{13}{|l|}{ Level } \\
\hline Slope & 0.56 & {$[0.38,0.84]$} & 0.005 & 0.71 & {$[0.46,1.09]$} & 0.112 & 0.48 & {$[0.31,0.75]$} & 0.001 & & & \\
\hline Curvature & & & & 1.36 & {$[0.93,1.00]$} & 0.112 & & & & & & \\
\hline Volatility & & & & 1.85 & {$[1.19,2.89]$} & 0.007 & 1.57 & {$[0.95,2.59]$} & 0.077 & & & \\
\hline $\begin{array}{l}\text { Baseline symptom } \\
\text { level }\end{array}$ & 2.15 & {$[1.38,3.37]$} & 0.001 & & & & 1.85 & {$[1.17,2.92]$} & 0.009 & & & \\
\hline \multicolumn{13}{|l|}{ Craving } \\
\hline Level & 0.66 & {$[0.44,1.00]$} & 0.048 & 0.72 & {$[0.49,1.06]$} & 0.092 & 0.60 & {$[0.39,0.92]$} & 0.018 & & & \\
\hline \multicolumn{13}{|l|}{ Slope } \\
\hline \multicolumn{13}{|l|}{ Curvature } \\
\hline Volatility & 0.69 & {$[0.47,1.03]$} & 0.072 & 0.68 & {$[0.45,1.02]$} & 0.064 & 0.64 & {$[0.42,1.00]$} & 0.047 & & & \\
\hline Age & 1.05 & {$[1.00,1.11]$} & 0.062 & 1.04 & {$[0.99,1.09]$} & 0.085 & 1.06 & {$[1.01,1.12]$} & 0.024 & & & \\
\hline FTND & & & & & & & 0.81 & {$[0.66,1.00]$} & 0.051 & & & \\
\hline
\end{tabular}

Odds ratios are shown for the variables retained in the final backward stepwise models. The levels of statistical significance for variable entry and removal were set at 0.05 and 0.10 , respectively. Odds ratios are expressed per standard deviation change in the trajectory parameters. Covariates were gender, age, FTND score, and treatment (nicotine vs placebo patch) and the baseline value for the withdrawal symptom component $P O M S$ the Profile of Mood States, SWQ Shiffman Withdrawal Questionnaire, FTND Fagerström Test of Nicotine Dependence

${ }^{a}$ Single-component regression refers to regressions that each include trajectory parameters of a single symptom component as primary independent variables

${ }^{b}$ Joint analysis regression refers to the combination of trajectory parameters of a subtype of negative affect (tension-anxiety, depression, or anger) and those of craving as primary independent variables in each stepwise logistic regression 
Fig. 1 Observed (black lines) and fitted (gray lines) trajectories of affective symptoms and craving across the initial 44-day tobacco abstinence. The left panels depict the mean trajectories of POMS TensionAnxiety (a), Depression (c), Anger (e), and SWQ Craving scores $(\mathbf{g})$ in the abstainers $(N=52 ;$ squares $)$ and relapsers $(N=88$; circles $)$ at 3 months postquit. The right panels $(\mathbf{b}, \mathbf{d}, \mathbf{f}$, and $\mathbf{h}$ ) show the trajectories of those measurements in the abstainers ( $N=19$; squares $)$ and relapsers $(N=121$; circles $)$ at 1 year postquit. Group means for each time point were derived from postcessation symptom levels that were each normalized to the baseline values for individual subjects. Fitted curves were obtained from the estimated trajectory parameters (i.e., level, slope, and curvature) from 2-level HLM modeling with Level 1 submodel, representing the trajectory of each symptom component, and Level 2 submodel, including abstinence status at 3 months or 1 year as a predictor (gray continuous lines, abstainers; gray dash lines, relapsers)
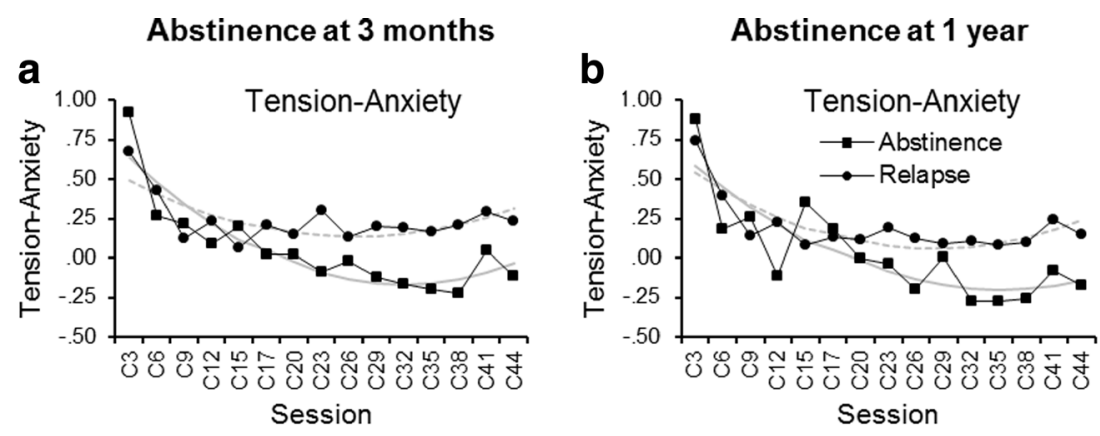

C

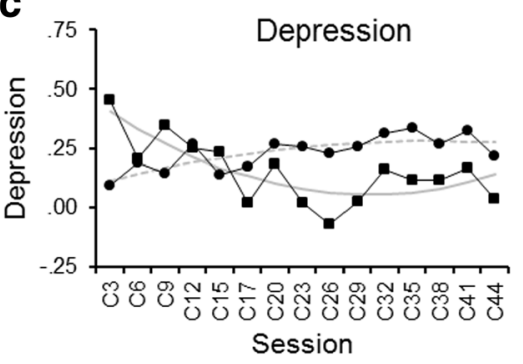

d

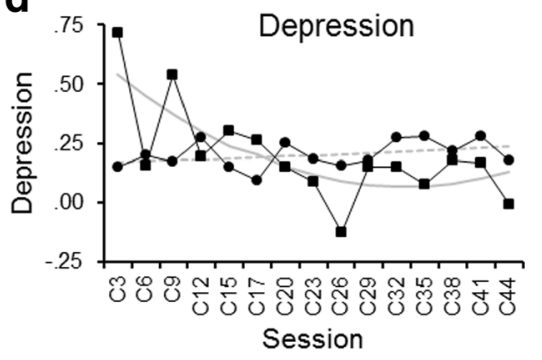

e

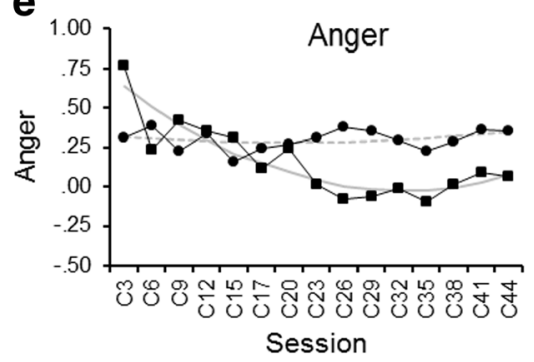

f

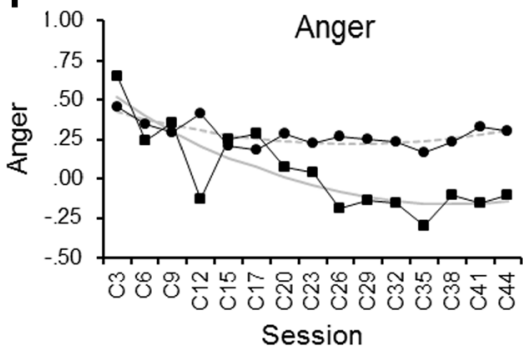

h

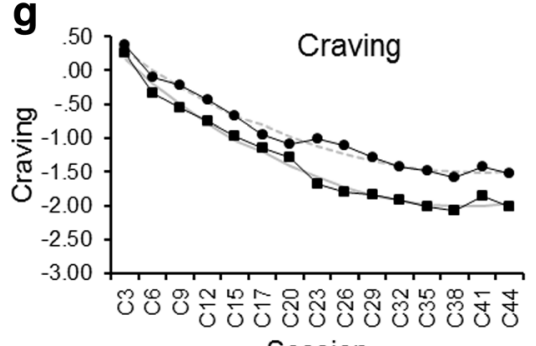

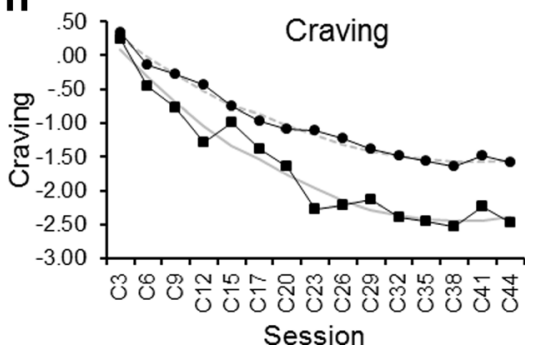

Results from the regression including only trajectory parameters of the SWQ craving scores as primary independent variables indicated that higher levels of postquit craving were marginally predictive of lower odds of the 3-month abstinence $(p=0.07$; Fig. $1 \mathrm{~g})$. Joint regression analysis was then conducted to assess whether trajectory parameters of any of the NA components independently predict abstinence with the parameters of the craving scores entered in the model.

As summarized in the bottom section of Table 2, trajectory profiles of the SWQ craving scores remained to be predictive of the 3-month abstinence in the joint regressions. Higher levels of craving were associated with lower odds of abstinence in the joint analysis with trajectory parameters of POMS tension-anxiety and anger and, marginally, depression scores ( $p=0.048, p=0.018, p=0.092$, respectively). There were also trends for greater volatility of the craving scores to be predictive of lower odds of the 3-month abstinence in the regressions including trajectory parameters of either tensionanxiety, anger, or depression scores $(p=0.072, p=0.064$, $p=0.047$, respectively). With these trajectory parameters for craving in the models, some trajectory parameters of the NA symptoms remained significant predictors of the 3-month cessation outcomes. Similar to results from the regressions with trajectory parameters of a single symptom component, the values of slope of tension-anxiety and anger trajectories were predictive of the 3-month abstinence ( $p=0.005, p=0.001$, respectively) in the joint regressions. The volatility in depression and to the marginally significant level, anger symptoms, 

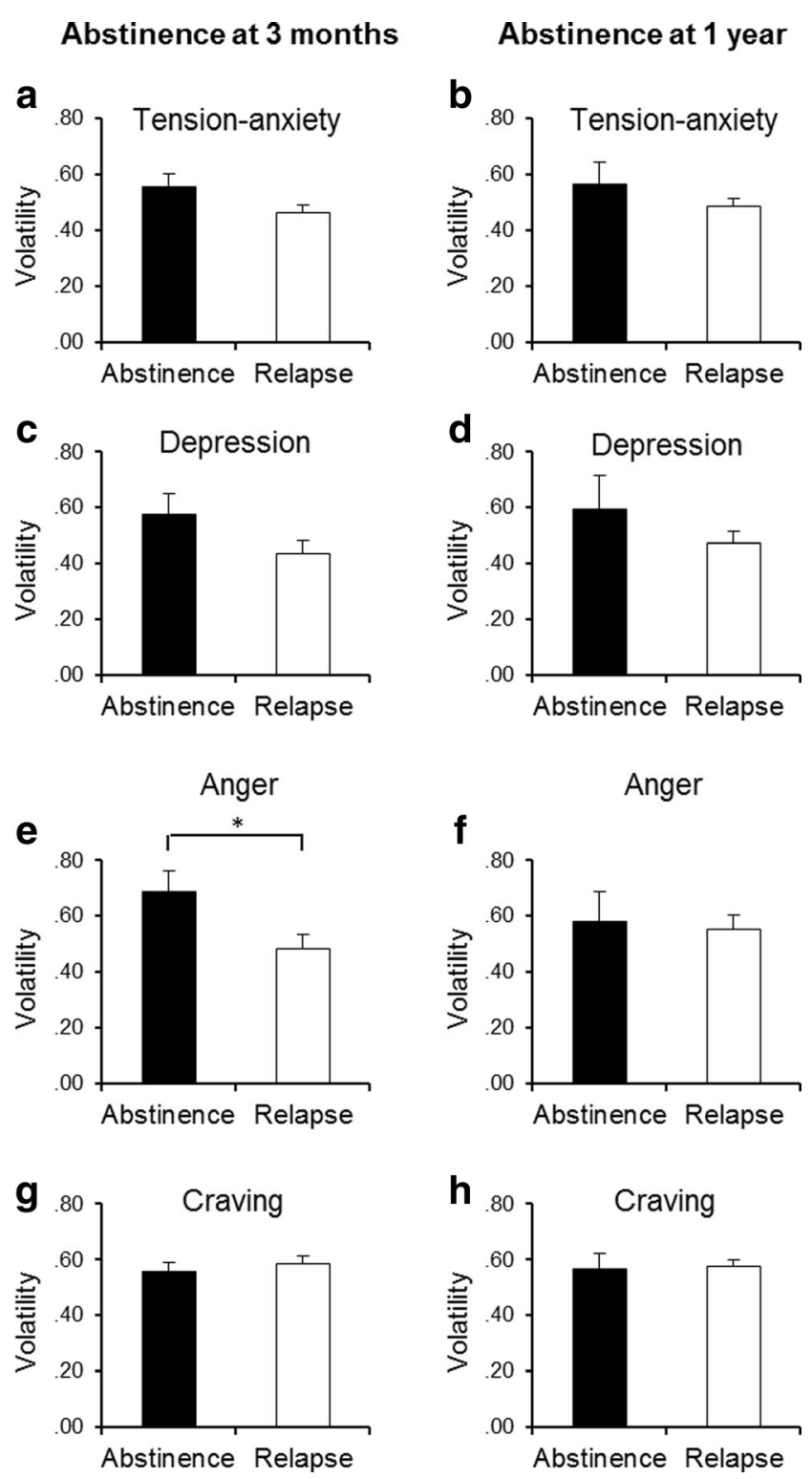

Fig. 2 Volatility of affective symptoms and craving across the initial 44day tobacco abstinence. The left panels depict the mean volatility of POMS Tension-Anxiety (a), Depression (c), Anger (e), and SWQ Craving scores $(\mathbf{g})$ in the abstainers $(N=52$; filled $)$ and relapsers $(N=88 ;$ blank $)$ at 3 months postquit. The right panels $(\mathbf{b}, \mathbf{d}, \mathbf{f}$, and $\mathbf{h})$ show the mean volatility of those measurements in the abstainers $(N=19$; filled $)$ and relapsers $(N=121$; blank $)$ at 1 year postquit. Group mean for each symptom component was derived from person-specific volatility parameters which were calculated as the root mean square differences between the observed symptom scale scores and the predicted scores from fitted trajectory parameters. ${ }^{*} p<.05$ ( $t$ test)

were also associated with the abstinence outcome $(p=0.007$ and $p=0.077$, respectively). Additionally, greater prequit baseline severity of tension-anxiety and anger were also predictive of increased odds of 3-month abstinence independent of the predictive ability of the craving symptoms. Among other control variables, age tended to be a predictor of cessation outcomes with older ages related to greater odds of abstinence across the joint regressions. In the joint regression with the trajectory parameters of anger symptoms and craving as primary variables, there was a tendency for higher FTND scores to be linked to lower odds of abstinence at 3 months. However, neither treatment (nicotine vs placebo patch) nor gender was a significant predictor of the 3-month abstinence in any of the regressions (omitted from Table 2).

\section{Prediction of abstinence at 1 year}

The top section of Table 3 summarizes the odds ratios of abstinence at 1 year postquit as significantly predicted by trajectory parameters of a symptom subtype in logistic regression analysis. Similar to the finding from the regressions with the 3-month abstinence as the dependent variable, the values of slope of POMS depression, anger, and marginally, tensionanxiety symptom trajectories were predictive of 1 year abstinence ( $p=0.047, p=0.012$, and $p=0.057$, respectively). As shown in Fig. 1b, d, and f), a greater decline in these NA symptoms over the treatment period was seen among those achieving 1 year abstinence as compared to the relapsers. Higher levels of the anger symptom trajectory were also associated with lower odds of 1 year abstinence $(p=0.043$; Fig. 1f). Both level and slope of the changes in SWQ craving score were predictors of the 1 year cessation outcome ( $p=0.014$ and $p=0.057$, respectively). As compared to the relapsers, those achieving 1 year abstinence reported generally lower craving and greater decline of the craving symptoms over the first 44 days following cessation (Fig. 1f).

Results from the joint regressions are presented in the bottom section of Table 3. Higher levels of craving remained to be predictive of lower odds of 1 year abstinence in the joint analysis with trajectory parameters of either POMS tensionanxiety, depression, or anger scores $(p=0.012, p=0.014$, and $p=0.015$, respectively). In the joint analysis with the trajectory profiles of depression scores, the values of slope of craving were also marginally associated with 1 year abstinence $(p=0.057)$. Apart from the predictive associations of trajectory profiles of craving and the cessation outcomes, the values of slope of the tension-anxiety and anger symptoms remained significant predictors in the respective joint regressions $(p=0.041$ and $p=0.035)$. However, none of the trajectory parameters of depressive scores was predictive of 1 year abstinence after adjustment for the effects of the trajectory level and slope of craving. Among the control variables, FTND scores tended to be a predictor of cessation outcomes with higher scores related to lowers odds of abstinence across the regressions. In addition, older ages tended to be associated with greater odds of abstinence. However, neither treatment (nicotine vs placebo patch) nor gender was a significant predictor of 1 year abstinence in any of these regressions (omitted from Table 3). 
Table 3 Relationships between withdrawal symptom trajectory parameters and abstinence at 1 year postquit in stepwise regressions

\begin{tabular}{|c|c|c|c|c|c|c|c|c|c|c|c|c|}
\hline & \multicolumn{3}{|c|}{ POMS Tension-anxiety } & \multicolumn{3}{|c|}{ POMS depression } & \multicolumn{3}{|c|}{ POMS anger } & \multicolumn{3}{|c|}{ SWQ craving } \\
\hline & $\begin{array}{l}\text { Odds } \\
\text { ratio }\end{array}$ & $\begin{array}{l}\text { 95\% C.I. } \\
\text { for odds ratio }\end{array}$ & $\begin{array}{l}p \\
\text { value }\end{array}$ & $\begin{array}{l}\text { Odds } \\
\text { ratio }\end{array}$ & $\begin{array}{l}95 \% \text { C.I. } \\
\text { for odds ratio }\end{array}$ & $\begin{array}{l}p \\
\text { value }\end{array}$ & $\begin{array}{l}\text { Odds } \\
\text { ratio }\end{array}$ & $\begin{array}{l}95 \% \text { C.I. } \\
\text { for odds ratio }\end{array}$ & $\begin{array}{l}p \\
\text { value }\end{array}$ & $\begin{array}{l}\text { Odds } \\
\text { ratio }\end{array}$ & $\begin{array}{l}95 \% \text { C.I. } \\
\text { for odds ratio }\end{array}$ & $\begin{array}{l}p \\
\text { value }\end{array}$ \\
\hline \multicolumn{13}{|c|}{ Single-component regression ${ }^{\mathrm{a}}$} \\
\hline Level & & & & & & & 0.51 & {$[0.27,0.98]$} & 0.043 & 0.46 & {$[0.24,0.85]$} & 0.014 \\
\hline Slope & 0.62 & {$[0.37,1.01]$} & 0.057 & 0.58 & {$[0.34,0.99]$} & 0.047 & 0.44 & {$[0.23,0.83]$} & 0.012 & 0.60 & {$[0.36,1.02]$} & 0.057 \\
\hline \multicolumn{13}{|c|}{$\begin{array}{l}\text { Curvatu- } \\
\text { re }\end{array}$} \\
\hline \multicolumn{13}{|c|}{ Volatility } \\
\hline Age & & & & & & & & & & 1.06 & {$[1.00,1.13]$} & 0.060 \\
\hline FTND & 0.82 & {$[0.64,1.05]$} & 0.109 & 0.81 & {$[0.64,1.03]$} & 0.087 & 0.77 & {$[0.60,1.00]$} & 0.050 & 0.78 & {$[0.59,1.02]$} & 0.067 \\
\hline \multicolumn{13}{|c|}{ Joint analysis regression $^{\mathrm{b}}$} \\
\hline \multicolumn{13}{|l|}{ Affect } \\
\hline \multicolumn{13}{|l|}{ Level } \\
\hline Slope & 0.56 & {$[0.32,0.98]$} & 0.041 & & & & 0.55 & {$[0.31,0.96]$} & 0.035 & & & \\
\hline \multicolumn{13}{|c|}{$\begin{array}{l}\text { Curvatu- } \\
\text { re }\end{array}$} \\
\hline & & & & & & & & & & & & \\
\hline \multicolumn{13}{|c|}{$\begin{array}{l}\text { Volatility } \\
\text { Craving }\end{array}$} \\
\hline Level & 0.47 & {$[0.26,0.87]$} & 0.015 & 0.46 & {$[0.24,0.85]$} & 0.014 & 0.47 & {$[0.26,0.86]$} & 0.015 & & & \\
\hline Slope & & & & 0.60 & {$[0.36,1.02]$} & 0.057 & & & & & & \\
\hline \multicolumn{13}{|l|}{ Curvature } \\
\hline \multicolumn{13}{|c|}{ Volatility } \\
\hline Age & 1.06 & {$[1.00,1.13]$} & 0.059 & 1.06 & {$[1.00,1.13]$} & 0.060 & 1.07 & {$[1.00,1.14]$} & 0.054 & & & \\
\hline FTND & 0.74 & {$[0.56,0.98]$} & 0.037 & 0.78 & {$[0.59,1.02]$} & 0.067 & 0.73 & {$[0.55,0.97]$} & 0.027 & & & \\
\hline
\end{tabular}

Odds ratios are shown for the variables retained in the final backward stepwise models. The levels of statistical significance for variable entry and removal were set at 0.05 and 0.10 , respectively. Odds ratios are expressed per standard deviation change in the trajectory parameters. Covariates were gender, age, FTND score, and treatment (nicotine vs placebo patch) and the baseline value for the withdrawal symptom component $P O M S$ the Profile of Mood States, SWQ Shiffman Withdrawal Questionnaire, FTND Fagerström Test of Nicotine Dependence

${ }^{a}$ Single-component regression refers to regressions that each include trajectory parameters of a single symptom component as primary independent variables

${ }^{\mathrm{b}}$ Joint-analysis regression refers to the combination of trajectory parameters of a subtype of negative affect (tension-anxiety, depression, or anger) and those of craving as primary independent variables in each stepwise logistic regression

\section{Prediction of abstinence at 6 and 9 months}

Additional regression analyses were conducted to evaluate the predictive associations of affective symptoms and craving with abstinence status at 6 and 9 months postquit. The levels of statistical significance for variable removal were set at 0.20 to allow variables with a trend towards significance to be retained in the models. As summarized in Supplemental Table S2 and S3 and illustrated in Fig. S3, greater values of level and slope of SWQ craving were predictive of lower odds of abstinence at both of these intermediate time points in the final regression models regardless of whether trajectory parameters of any of the affective symptom components were included in the initial models. In regression models with trajectory parameters of a single symptom component, the trajectory features of POMS anger scores tended to be associated with abstinence at 6 months (level) and at 9 months (level and slope). There were tendencies for slopes of POMS tension-anxiety to predict abstinence at both time points and for curvature coefficients and slopes of depression scores to predict abstinence at 6 and 9 months, respectively. Unlike the results for prediction of abstinence at 3 months and
1 year, trajectory parameters for none of the affective symptom components were significant predictors of abstinence at 6 or 9 months, independent of level and slope of craving, in the final models of joint regression.

\section{Discussion}

The current findings that smaller reductions in anxiety and anger symptoms across the first 44 days of abstinence were predictive of greater relapse risk at 3 months and 1 year, even after controlling for the effects of craving, provide support for the primary study hypotheses. These results also highlight differences between the specific NA subtypes in their ability to predict relapse and suggest the value of incentivized minimization of relapse during the first weeks of abstinence when assessing the impact of NA symptom trajectories on subsequent relapse. Our results contrast with those of Javitz et al. (2012) who found that craving trajectories (level, slope, and volatility), but not NA trajectories, over the first 7 days postquit to predict abstinence status at 8 weeks and 6 months. The reason for these differences between studies may relate to 
our use of a novel relapse-minimization procedure across the first 45 days of abstinence and the use of extended, 44-day symptom trajectories as predictors. While previous studies using singleoccasion measurements have found inconsistent associations between specific affective withdrawal symptoms and subsequent poorer smoking cessation outcomes (cf. Hughes 2007), the present study demonstrates that slower dissipation of anger and anxiety symptoms over the first 44 days of abstinence is a potent predictor of relapse.

Taken together, the current findings are congruent with and expand the data from two previous studies (Piasecki et al. 2000; Piper et al. 2011) that demonstrated NA and craving to be two major components of tobacco withdrawal that each uniquely predict relapse. Collectively, these data support integrative theories of drug addiction that account for the link of the two components to relapse vulnerability. For instance, the elaborated intrusion theory of craving by Kavanagh et al. (2005) has proposed that while craving is a strong motivating force, it is only one of the many factors that influence drug seeking and drug use. Among these factors, NA can trigger, intensify craving, and also modulate the relationship between craving and drug use through other cognitive-behavioral mechanisms (e.g., impairing self-efficacy in behavioral control). Consistent with this notion, there were overall modest but significant correlations between trajectories of the NA symptoms and those of craving during the 44day early abstinence (see Supplemental Table S1).

Although trajectory parameters of the three major subtypes of NA symptoms were moderately correlated (see Supplemental Table S1), the present analysis indicated that these components might differ in their association with subsequent risk for relapse. After controlling for the effects of craving, the trajectory profiles, primarily the slope, and of tension-anxiety and anger symptoms remained predictive of abstinence at 3 months and 1 year, whereas those of depressive symptoms exhibited no significant associations with the 1-year cessation outcome and overall weak associations (except for volatility) with the 3-month outcome (Table 2). Thus, the results suggest that the link between depressive symptoms and relapse is significantly mediated by craving, whereas the symptoms of anger and anxiety and relapse are linked to relapse in ways largely independent of craving. Part of these findings is in accord with a previous clinical report that craving mediated the relation between depressive symptoms and relapse rates among substance users (Witkiewitz and Bowen 2010). The present results are also congruent with findings that high-arousal NA subtypes (e.g., anger and anxiety) versus low-arousal NAs (e.g., depression) appear to be stronger mediators of the relation of abstinence and reinstatement of smoking in laboratory settings (Aguirre et al. 2015). Future research should continue to examine likely differences among the subtypes of affective symptoms with respect to their direct and/or indirect impact on relapse risks.
The mechanisms underlying the observed links between trajectories of affective symptoms and relapse risk remain poorly characterized. The persistence of affective symptoms among individuals at higher risk for relapse could reflect a combination of smoking- and abstinence-related long-lasting neuropharmacological and biological processes (e.g., Cosgrove et al. 2009; Gilbert et al. 1999; Gilbert et al. 2003; Koob et al. 2014), environmental events (e.g., stressors or conditioned stimuli; Piasecki et al. 2003a, b), and person variables (e.g., genes and affective traits; Gilbert et al. 2009). One plausible explanation for the main findings of the current analysis is that refractory or slower decreases in anxiety and anger symptoms reflect persistent, possibly genetically influenced, dysregulations in brain stress response systems (Koob et al. 2014), and/or unmasking of dispositional negative affect (Gilbert, 1995) that contribute to the risk for relapse in addition to the vulnerability associated with sensitized brain reward systems (Robinson and Berridge 1993) as manifested in the profiles of craving during early weeks of abstinence. An alternative, but not mutually exclusive, possibility is that greater decline of affective symptoms during early abstinence in some individuals results in greater enhancement of selfefficacy in coping with stressors that often precipitate relapse which in turn contributes to higher success rates of long-term cessation (Hendricks et al. 2010; Marlatt and George 1984). Future investigations should evaluate these possibilities and other potential mechanisms for the link between the trajectories of affective symptoms and cessation outcome.

Interestingly, in contrast to an earlier report of an association of greater volatility of general abstinence symptoms with lapses (Piasecki et al., 2003a), volatility of NA was generally not related to relapse, and when it was the direction was in the opposite of the predicted direction, greater volatility of changes in post-cessation anger, and, to a marginally significant level, depression scores were also related to greater odds of the 3-month abstinence. It is possible that incentives for abstinence may have led to either increased or reduced affective volatility because of incentive-induced commitment to remain abstinent. On the other hand, the current findings are partially consistent with Javitz et al. (2012) who observed a trend for the link between greater volatility of NA and higher abstinence rates at 6 months postquit. Future research is needed to reconcile these seemingly inconsistent results.

The present findings may have important clinical implications. First, as the profiles of affective symptoms, particularly those of anxiety and anger, are linked to long-term cessation outcome largely independent of the mediational effects of craving, reduction in these NA states should be a valid target for pharmacological and psychosocial interventions to improve the long-term efficacy of smoking cessation. Moreover, both the profiles of affective symptoms and those of craving over the early weeks of abstinence appear to have prognostic validity. Individualized strategies 
of cessation treatment and/or relapse prevention are particularly needed for those individuals with refractory symptoms of anxiety or anger. Given that current pharmacological treatments may have limited effects on anxiety and anger symptoms (Cinciripini et al. 2013), our findings highlight a possibility that new treatments could lead to improved cessation outcome by reducing NA and diminishing its motivational drive for relapse to smoking.

Some limitations of this study should be noted. First, by excluding the small number of individuals who relapsed during the 45-day treatment period, the present analysis might have underestimated the predictive values of trajectory features (e.g., slope or curvature) reflecting severity of early withdrawal symptoms which were likely to be greater in those relapsed before the end of treatment. Second, inclusion of symptom measurements at finer time points within the first 1-2 days after cessation might have been more informative concerning the likely contribution of early, presumably intense, affective and craving symptoms to the prediction of later relapse likelihood. Third, reliance on retrospective self-ratings of average symptom severity over the past 3 days might introduce recollection biases and render the ratings less sensitive to transient, situational changes in the abstinence symptoms than real-time reports such as ecological momentary assessment (Shiffman et al. 2008). Fourth, the monetary incentives may have influenced the participants' ratings of their symptoms in subtle and complicated ways (e.g., cognitive dissonance processes). Finally, statistically significant associations between trajectory parameters of tension-anxiety and anger symptoms and abstinence status while controlling for the effects of craving were observed for outcomes at 3 months and 1 year, but not those at 6 and 9 months. It is unclear whether limited sample size (Vittinghoff and McCulloch 2007) or other factors (e.g., unassessed negative life events, negative affect, or smoking cue exposure) contributed to this temporal discrepancy.

In summary, this study has shown that temporal profiles of affective symptoms, especially the trends of decline in anxiety and anger, during the early weeks of abstinence, are predictive of cessation outcome at 3 months and 1 year postquit, independent of the predictive ability of craving. These results provide new evidence for the motivational significance of negative affect in relapse to smoking and the validity of negative affect reduction as an important target for smoking cessation interventions. Finally, it should be noted that it is generally assumed that one of the effects of NA is to increase craving (Dziak et al. 2015). Thus, statistically controlling for craving when examining NA-withdrawal association may have resulted in an underestimation of important variance accounted for by affective symptoms.
Acknowledgments The authors thank GlaxoSmithKline for providing nicotine and placebo patches used in this study.

\section{Compliance with ethical standards}

Conflict of interest Yantao Zuo, Norka E. Rabinovich, and David G Gilbert declare that they have no conflict of interest.

Funding This study was funded by the National Institute on Drug Abuse (Grant R01 DA12289).

\section{References}

Aguirre CG, Madrid J, Leventhal AM (2015) Tobacco withdrawal symptoms mediate motivation to reinstate smoking during abstinence. J Abnorm Psychol 124(3):623-634. doi:10.1037/abn0000060

Baker TB, Breslau N, Covey L, Shiffman S (2012) DSM criteria for tobacco use disorder and tobacco withdrawal: a critique and proposed revisions for DSM-5. Addiction 107(2):263-275. doi:10.1111/j.1360-0443.2011.03657.x

Baker TB, Piper ME, McCarthy DE, Majeskie MR, Fiore MC (2004) Addiction motivation reformulated: an affective processing model of negative reinforcement. Psychological Rev 111(1):33-51. doi:10.1037/0033-295X.111.1.33

Borland R, Partos TR, Yong HH, Cummings KM, Hyland A (2012) How much unsuccessful quitting activity is going on among adult smokers? Data from the international tobacco control four country cohort survey. Addiction 107(3):673-682

Castro Y, Kendzor DE, Businelle MS, Mazas CA, Cofta-Woerpel L, Cinciripini PM, Wetter DW (2011) Structural and predictive equivalency of the Wisconsin smoking withdrawal scale across three racial/ethnic groups. Nicotine Tob Res 13(7):548-555. doi:10.1093/ntr/ntr039

Cinciripini PM, Robinson JD, Karam-Hage M, Minnix JA, Lam C, Versace F, Brown VL, Engelmann JM, Wetter DW (2013) Effects of varenicline and bupropion sustained-release use plus intensive smoking cessation counseling on prolonged abstinence from smoking and on depression, negative affect, and other symptoms of nicotine withdrawal. JAMA Psychiatry 70(5):522-533. doi:10.1001/jamapsychiatry.2013.678

Cofta-Woerpel L, McClure JB, Li Y, Urbauer D, Cinciripini PM, Wetter DW (2011) Early cessation success or failure among women attempting to quit smoking: trajectories and volatility of urge and negative mood during the first postcessation week. J Abnorm Psychol 120(3):596-606. doi:10.1037/a0023755

Cook J, Spring B, McChargue D, Doran N (2010) Effects of anhedonia on days to relapse among smokers with a history of depression: a brief report. Nicotine Tob Res 12(9):978-982. doi:10.1093 /ntr/ntq118

Cosgrove KP, Batis J, Bois F, Maciejewski PK, Esterlis I, Kloczynski T, Stiklus S, Krishnan-Sarin S, O'Malley S, Perry E, Tamagnan G, Seibyl JP, Staley JK (2009) ß2-nicotinic acetylcholine receptor availability during acute and prolonged abstinence from tobacco smoking. Arch Gen Psychiatry 66(6):666-676

Daughton DM, Roberts D, Patil KD, Rennard SI (1990) Smoking cessation in the workplace: evaluation of relapse factors. Prev Med 19(2): $227-230$

Dziak JJ, Li R, Tan X, Shiffman S, Shiyko MP (2015) Modeling intensive longitudinal data with mixtures of nonparametric trajectories and 
time-varying effects. Psychol Methods 20(4):444-469. doi:10.1037 met0000048

Etter JF, Hughes JR (2006) A comparison of the psychometric properties of three cigarette withdrawal scales. Addiction 101(3):362-372

Gilbert DG (1995) Smoking: individual differences, psychopathology, and emotion. Taylor \& Francis, Washington

Gilbert DG, McClernon FJ, Rabinovich NE, Dibb WD, Plath LC, Hiyane S, Jensen RA, Meliska CJ, Estes SL, Gehlbach BA (1999) EEG, physiology, and task-related mood fail to resolve across 31 days of smoking abstinence: relations to depressive traits, nicotine exposure, and dependence. Exp Clin Psychopharmacol 7(4):427-443

Gilbert DG, Zuo Y, Browning RA, Shaw TM, Rabinovich NE, GilbertJohnson AM, Plath L (2003) Platelet monoamine oxidase B activity changes across 31 days of smoking abstinence. Nicotine Tob Res 5(6):813-819

Gilbert DG, Zuo Y, Rabinovich NE, Riise H, Needham R, Huggenvik JI (2009) Neurotransmission-related genetic polymorphisms, negative affectivity traits, and gender predict tobacco abstinence symptoms across 44 days with and without nicotine patch. J Abnorm Psychol 118(2):322-334. doi:10.1037/a0015382

Heatherton TF, Kozlowski LT, Frecker RC, Fagerström KO (1991) The Fagerström test for nicotine dependence: a revision of the Fagerström tolerance questionnaire. Br J of Addict 86(9):1119 1127. doi:10.1111/j.1360-0443.1991.tb01879.x

Hendricks PS, Delucchi KL, Hall SM (2010) Mechanisms of change in extended cognitive behavioral treatment for tobacco dependence. Drug Alcohol Depend 109(1-3):114-119. doi:10.1016/j. drugalcdep.2009.12.021

Hughes JR (1992) Tobacco withdrawal in self-quitters. J Consult Clin Psychol 60(5):689-697

Hughes JR (2007) Effects of abstinence from tobacco: etiology, animal models, epidemiology, and significance: a subjective review. Nicotine Tob Res 9(3):329-339. doi:10.1080/14622200701188927

Hughes JR, Hatsukami D (1986) Signs and symptoms of tobacco withdrawal. Arch Gen Psychiatry 43(3):289-294

Hughes JR, Carpenter MJ, Naud S (2010) Do point prevalence and prolonged abstinence measures produce similar results in smoking cessation studies? A systematic review. Nicotine Tob Res 12(7): 756-762. doi:10.1093/ntr/ntq078

Jacob P III, Wilson M, Benowitz NL (1981) Improved gas chromatographic method for the determination of nicotine and cotinine in biologic fluids. J Chromatogr 222(1):61-70

Kavanagh DJ, Andrade J, May J (2005) Imaginary relish and exquisite torture: the elaborated intrusion theory of desire. Psychol Rev 112(2):446- 467

Koob GF, Buck CL, Cohen A, Edwards S, Park PE, Schlosburg JE, Schmeichel B, Vendruscolo LF, Wade CL, Whitfield TW Jr, George O (2014) Addiction as a stress surfeit disorder. Neuropharmacology 76(Pt B):370-382

Leventhal AM, Ramsey SE, Brown RA, LaChance HR, Kahler CW (2008) Dimensions of depressive symptoms and smoking cessation. Nicotine Tob Res 10(3):507-517. doi:10.1080 /14622200801901971

Marlatt GA, George WH (1984) Relapse prevention: introduction and overview of the model. Br J Addict 79(3):261-273

McCarthy DE, Piasecki TM, Fiore MC, Baker TB (2006) Life before and after quitting smoking: an electronic diary study. J Abnorm Psychol 115(3):454-466
McNair D, Lorr M, Droppelman D (1971) Profile of mood states. Educational and Industrial Testing Service, San Diego

Patten CA, Martin JE (1996) Does nicotine withdrawal affect smoking cessation? Clinical and theoretical issues. Ann Behav Med 18(3): 190-200. doi:10.1007/BF02883397

Patterson F, Kerrin K, Wileyto EP, Lerman C (2008) Increase in anger symptoms after smoking cessation predicts relapse. Drug Alcohol Depend 95(1-2):173-176. doi:10.1016/j.drugalcdep.2008.01.013

Piasecki TM (2006) Relapse to smoking. Clin Psychol Rev 26(2):196215. doi:10.1016/j.cpr.2005.11.007

Piasecki TM, Niaura R, Shadel WG, Abrams D, Goldstein M, Fiore MC, Baker TB (2000) Smoking withdrawal dynamics in unaided quitters. J Abnorm Psychol 109(1):74-86. doi:10.1037/0021-843 X.109.1.74

Piasecki TM, Jorenby DE, Smith SS, Fiore MC, Baker TB (2003a) Smoking withdrawal dynamics: I. Abstinence distress in lapsers and abstainers. J Abnorm Psychol 112:3-13

Piasecki TM, Jorenby DE, Smith SS, Fiore MC, Baker TB (2003b) Smoking withdrawal dynamics: II. Improved tests of withdrawalrelapse relations. J Abnorm Psychol 112:14-27

Piper ME, Schlam TR, Cook JW, Sheffer MA, Smith SS, Loh WY, Bolt DM, Kim SY, Kaye JT, Hefner KR, Baker TB (2011) Tobacco withdrawal components and their relations with cessation success. Psychopharmacol (Berl) 216(4):569-578. doi:10.1007/s00213-0112250-3

Raudenbush SW, Bryk AS (2002) Hierarchical linear models: applications and data analysis method (2nd ed.). Sage, Newbury Park

Robinson TE, Berridge KC (1993) The neural basis of drug craving: an incentive sensitization theory of addiction. Brain Res Rev 18:247291

Shiffman S (1979) The tobacco withdrawal syndrome. In: NA Krasnegor NA (ed) Cigarette smoking as a dependence process (NIDA research monograph no. 23). Public Health Service, Rockville, pp 153-184

Shiffman S, Stone AA, Hufford MR (2008) Ecological momentary assessment. Annu Rev Clin Psychol 4:1-32

Solomon RL (1977) An opponent-process theory of acquired motivation: the affective dynamics of addiction. In: Maser JD, Seligman MEP (eds) Psychopathology: experimental models. W. H. Freeman, San Francisco, pp 66-103

Vittinghoff E, McCulloch CE (2007) Relaxing the rule of ten events per variable in logistic and cox regression. Am J Epidemiol 165(6):710 718

Welsch SK, Smith SS, Wetter DW, Jorenby DE, Fiore MC, Baker TB (1999) Development and validation of the Wisconsin smoking withdrawal scale. Exp Clin Psychopharmacol 7(4):354-361

Witkiewitz K, Bowen S (2010) Depression, craving, and substance use following a randomized trial of mindfulness-based relapse prevention. J Consult Clin Psychol 78:362-374

Wray JM, Gass JC, Tiffany ST (2013) A systematic review of the relationships between craving and smoking cessation. Nicotine Tob Res 15(7):1167-1182. doi:10.1093/ntr/nts268

Zuo Y, Gilbert DG, Rabinovich NE, Riise H, Needham R, Huggenvik JI (2009) DRD2-related TaqIA polymorphism modulates motivation to smoke. Nicotine Tob Res 11(11):1321-1329. doi:10.1093 /ntr/ntp141 